05,13

\title{
Угловые зависимости спектров спин-волнового резонанса в неоднородных пленках с орторомбической анизотропией
}

\author{
(C) А.М. Зюзин, М.А. Бакулин, В.В. Радайкин, Н.В. Янцен \\ Мордовский государственный университет им. Н.П. Огарёва, \\ Саранск, Россия \\ E-mail: zyuzin.am@rambler.ru
}

(Поступила в Редакцию 23 июня 2016 г.)

\begin{abstract}
Обнаружено, что в неоднородных магнитных пленках с орторомбической анизотропией на двух из трех характерных угловых зависимостях резонансных полей высокоинтенсивных спин-волновых мод происходит смена области локализации нулевой моды. Показано, что по резонансным полям нулевой и последней из высокоинтенсивных спин-волновых мод спектров спин-волнового резонанса можно определить значения полей анизотропии на одной и другой поверхностях пленки.
\end{abstract}

DOI: 10.21883/FTT.2017.02.44045.257

\section{1. Введение}

Исследование особенностей спиновой динамки в диэлектрических магнитных пленках представляет несомненный научный и практический интерес, обусловленный перспективами применения данных материалов в устройствах спинтроники и магнетоэлектроники [1-3]. Спектры спин-волнового резонанса (СВР) в многослойных пленках с однородными слоями, и в частности их угловые зависимости, изучены в работах [4-6]. В [5-7] показано, что, когда закрепление спинов обусловлено динамическим механизмом, при изменении угла между постоянным внешним магнитным полем Н и нормалью к пленке, наблюдается сначала уменышение, а затем возрастание количества возбуждаемых спин-волновых (CB) мод. При этом, в случае малых значений параметра затухания в слоях, происходит изменение области возбуждения стоячих гармонических CB-мод. При перпендикулярной ориентации Н относительно плоскости пленки указанные моды возбуждаются в одном слое, при параллельной в другом. В пленках с диссипативным механизмом закрепления спинов спектр СВР является практически изотропным $[8,9]$. Это обусловлено тем, что область возбуждения СВ-мод при изменении ориентации Н относительно пленки остается неизменной и локализованной в слое с малым значением параметра затухания.

В рассмотренных случаях область возбуждения всех СВ-мод, начиная с нулевой, охватывает весь объем слоя возбуждения. Распределение резонансных полей СВ-мод подчиняется квадратичному закону, разность резонансных полей нулевой и $n$-й $\mathrm{CB}$-мод $H_{0}-H_{n} \propto n^{2}$. Зависимость относительной интенсивности СВ-мод от их номера $n$ описывается как $I_{n} / I_{0} \propto 1 / n^{2}-1 / n^{4}$ [6]. СВР в неоднородных одноосных пленках исследован в работах $[6,10,11]$.

Целью настоящей работы являлось исследование угловых зависимостей спектров СВР в неоднородных пленках, обладающих линейным распределением полей одноосной и ромбической компонент анизотропии по их толщине.

\section{2. Эксперимент}

Исследуемые пленки ферритов-гранатов были выращены методом жидкофазной эпитаксии на подложках из гадолиний-галлиевого граната с кристаллографической плоскостью (110). Состав пленок $(\mathrm{BiLuGd})_{3}(\mathrm{FeGa})_{5} \mathrm{O}_{12}$ задавался соответствующим составом раствора в расплаве и определялся методом рентгеновского энергодисперсионного микроанализа. Намагниченность насыщения $4 \pi M$ равнялась $115 \mathrm{G}$, толщина $h=1.6 \mu \mathrm{m}$, константа обменного взаимодействия $A$, которая определялась по температуре Кюри [12], была равна $1.23 \cdot 10^{-7} \mathrm{erg} \cdot \mathrm{cm}^{-1}$, значение обменной жесткости $D=(2 A / M)=27 \cdot 10^{-9} \mathrm{erg} \cdot \mathrm{cm}^{-1} \cdot \mathrm{G}^{-1}$. Близкое к линейному распределение полей одноосной $H_{k u}$ и ромбической $H_{k r}$ компонент анизотропии по толщине пленок достигалось плавным изменением температуры раствора в расплаве в процессе эпитаксиального роста. Такой характер распределения $H_{k u}$ и $H_{k r}$ подтверждает анализ трансформации спектров СВР, происходящей при послойном стравливании. Результаты этого анализа позволили установить, что поле $H_{k u}$ изменяется от 447 Ое вблизи подложки до -1050 Ое на свободной поверхности. Поле $H_{k r}$ соответственно от 293 Ое до -378 Ое. Регистрация спектров СВР производилась при комнатной температуре на радиоспектрометре PS100.X на частоте микроволнового поля $v=9.3 \mathrm{GHz}$. Установку азимутального и полярного углов, определяющих ориентацию постоянного магнитного поля относительно кристаллографических осей пленки, производили с помощью специального двухкоординатного гониометра. Геометрия регистрации угловых зависимостей приведена на рис. 1. 

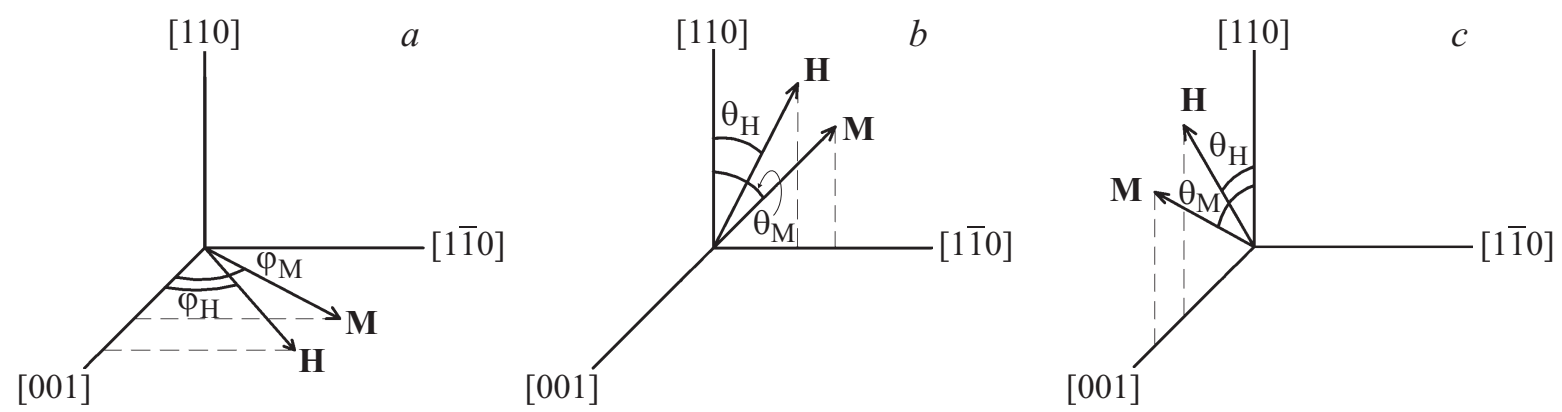

Рис. 1. Геометрия регистрации угловых зависимостей спектров СВР.

\section{3. Расчет}

Для расчета значений полей одноосной $H_{k u}$, ромбической $H_{k r}$ и кубической $H_{k 1}$ компонент анизотропии на одной и другой границах пленки нами использовались резонансные соотношения [13] для направлений Н: вдоль оси [110], совпадающей с нормалью к пленке,

$$
\left(\frac{\omega}{\gamma}\right)^{2}=\left(H_{1}+H_{k u}+\frac{H_{k 1}}{2}\right)\left(H_{1}+H_{k u}+H_{k r}-H_{k 1}\right),
$$

вдоль оси [1ํㅣㄹ в плоскости пленки, совпадающей с осью ромбической компоненты анизотропии

$$
\left(\frac{\omega}{\gamma}\right)^{2}=\left(H_{2}-H_{k r}+\frac{H_{k 1}}{2}\right)\left(H_{2}-H_{k u}-H_{k r}-H_{k 1}\right),
$$

и вдоль оси [001], также лежащей в плоскости пленки

$$
\left(\frac{\omega}{\gamma}\right)^{2}=\left(H_{3}-H_{k u}+H_{k 1}\right)\left(H_{3}+H_{k r}+H_{k 1}\right),
$$

где $H_{1}, H_{2}$ и $H_{3}$ - резонансные поля для соответствующих мод.

Расчет семейства кривых, соответствующих угловым зависимостям полей однородного резонанса для плоскостей пленки на различных расстояниях от подложки, производили с помощью соответствующих резонансных соотношений $[6,13]$ и условия равновесной ориентации намагниченности.

Расчет резонансных полей и интенсивностей пиков поглощения СВ-мод проводился путем решения волнового уравнения для переменной намагниченности [6]

$$
\frac{2 A}{M} \frac{\partial^{2} H}{\partial z^{2}}-\left(H+H_{k}^{\mathrm{eff}}-\frac{\omega}{\gamma}\right) m=0 .
$$

Здесь ось $z$ совпадает с нормалью к пленке. Эффективное поле анизотропии $H_{k}^{\text {eff }}$ учитывало вклад $H_{k u}$ и $H_{k r}$ и принималось линейно изменяющимся по толщине пленки $H_{k}^{\text {eff }}=B z+C$. Как показывает расчет, если $H_{k u}$ и $H_{k r}$ линейно изменяются по толщине, то распределение $H_{k}^{\mathrm{eff}}$, как и поля однородного резонанса $H_{u r}$, также будут близки к линейным по $z$. Поскольку значения $A$ и $M$ в существенно меньшей степени зависят от температуры роста, обменная жесткость $(2 A / M)$ при расчете принималась постоянной по толщине пленки.

С помощью замены переменной

$$
x=l^{-1}\left[z+\left(H+C-\frac{\omega}{\gamma}\right) B^{-1}\right],
$$

где $l=(2 A / M B)^{1 / 3}$, уравнение (4) приводили к виду $\frac{\partial^{2} m(x)}{\partial x^{2}}-x \cdot m(x)=0$. Решение данного уравнения представляет собой линейную комбинацию функций Эйри $m(x)=a \cdot A i(x)+b \cdot B i(x)$. Константы $a$ и $b$ находились путем учета граничных условий на поверхностях пленки $m^{\prime}\left(x_{0}\right)=m^{\prime}\left(x_{L}\right)=0$, а также условия нормировки $\int_{0}^{L} d z m^{2}(z)=1\left[x_{L} m^{2}\left(x_{L}\right)-x_{0} m^{2}\left(x_{0}\right)\right]=1$.

\section{4. Обсуждение результатов}

К одной из характерных особенностей спектров СВР в пленках с однородным градиентом эффективного поля анизотропии относится следующая. В отличие от отмеченных выше пленок с однородными слоями, в пленках с линейным изменением поля однородного резонанса по толщине зависимость интенсивности пиков возбуждаемых CB-мод от их номера можно разделить на два участка: 1 - участок высокоинтенсивных мод с близкими значениями амплитуд и 2 - участок СВ-мод с весьма малой интенсивностью. Первый участок соответствует возбуждению локализованных мод, для которых точка поворота $[14,15]$, соответствующая толщине, на которой волновое число СВ-моды переходит от действительных значений к мнимым, лежит в области пленки. Второй участок соответствует объемным модам, для которых волновое число является действительным на всей толщине пленки.

На рис. 2, a (слева) приведена экспериментальная угловая зависимость (азимутальная) $H_{n}\left(\varphi_{H}\right)$ резонансных полей высокоинтенсивных СВ-мод от угла $\varphi_{H}$ между $\mathbf{H}$ и осью [100], лежащих в плоскости пленки. На рис. 2, $b$ приведена угловая зависимость (полярная) резонансных полей СВ-мод $H_{n}\left(\theta_{H}\right)$ в плоскости $(100)$, перпендикулярной плоскости пленки. Угол $\theta_{H}$, определяющий направление внешнего поля $\mathbf{H}$, отсчитывался от нормали 

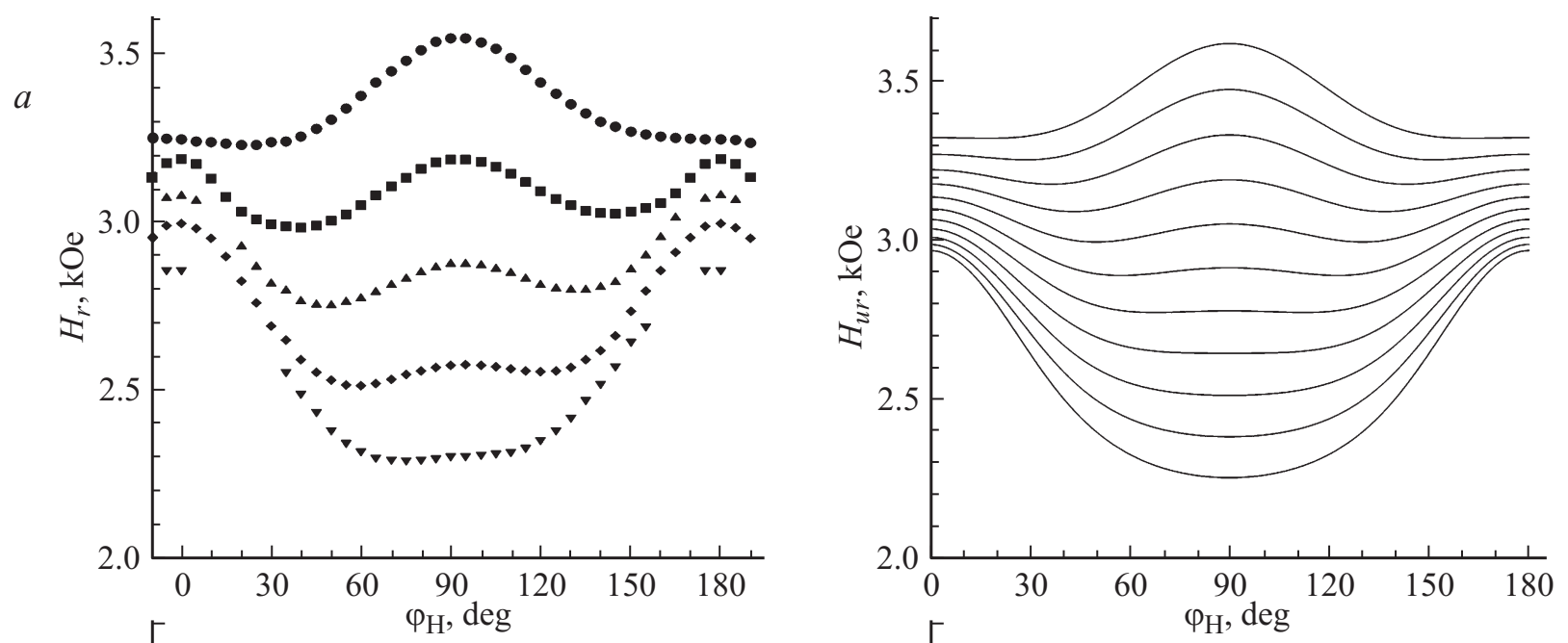

$b$
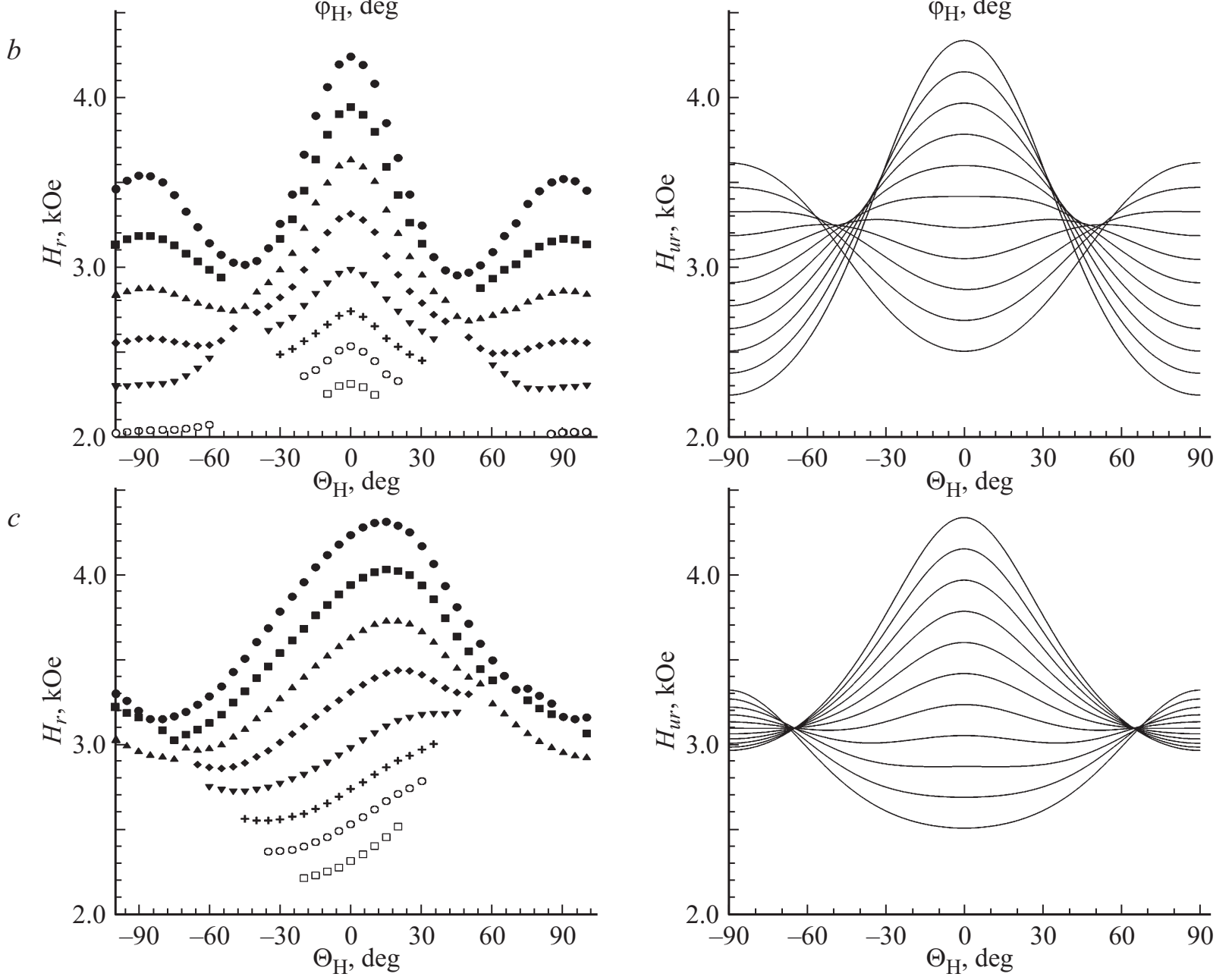

Рис. 2. Угловые зависимости резонансных полей СВ-мод (слева) и полей однородного резонанса для точек пленки на различном расстоянии от подложки (справа).

к пленке, совпадающей с осью [110]. Полярная угловая зависимость резонансных полей в плоскости (1ㅣㅣ), перпендикулярной плоскости пленки, приведена на рис. 2, c. Справа приведены соответствующие расчетные угловые зависимости полей однородного резонанса для точек пленки на различных расстояниях от подложки.
Трансформация спектра в зависимости от угла $\theta_{H}$ в плоскости (100) приведена на рис. 3. Как видно из рис. 2, угловые зависимости резонансных полей СВ-мод в пленках с орторомбической анизотропией, в отличие от двухслойных пленок с однородными слоями, имеют более сложный характер. В частности на полярных 


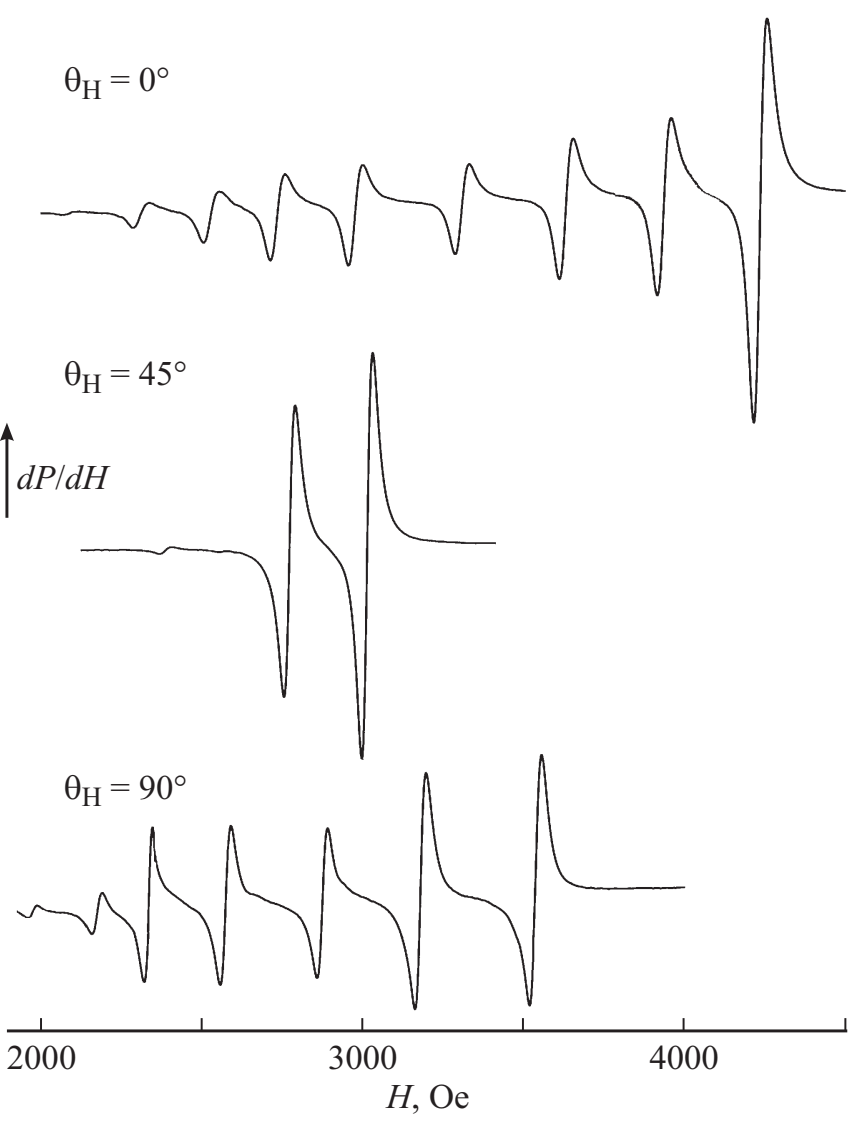

Рис. 3. Спектры СВР при различных ориентациях Н относительно нормали к пленке в плоскости (100).

зависимостях $H_{n}\left(\theta_{H}\right)$ при некоторых значениях $\theta_{H}$ происходит как бы их пересечение. Отметим, что нулевая (самая высокополевая) CВ-мода возбуждается в области с наибольшим для данной ориентации $\mathbf{H}$ значением поля однородного резонанса. Из рис. $2, b, c$ видно, что на полярной угловой зависимости резонансное поле нулевой моды плавно как бы „переходит“ в резонансное поле последней из высокоинтенсивных мод. И наоборот, в области, поле однородного резонанса которой близко к резонансному полю последней из высокоинтенсивных CB-мод при перпендикулярной ориентации, начинает возбуждаться нулевая СВ-мода. Данную особенность можно объяснить следующим образом. Наличие неоднородности в распределении полей одноосной и ромбической компонент анизотропии по толщине пленки приводит к различию в характере распределения полей однородного резонанса в зависимости от ориентации внешнего магнитного поля $\mathbf{H}$ относительно пленки. Это иллюстрируют рисунки справа, где приведены расчетные угловые зависимости полей однородного резонанса $H_{u r}\left(\theta_{H}\right)$, на которых также наблюдается пересечение зависимостей $H_{u r}$. Видно, что имеет место хорошее соответствие между экспериментальными угловыми зависимостями резонансных полей СВ-мод и соответствующими угловыми зависимостями полей однородного ре- зонанса для точек пленки на различных расстояниях от подложки. Некоторая асимметрия зависимостей $H_{n}\left(\theta_{H}\right)$ для плоскости (11̄0) объясняется отклонением („завалом“) оси одноосной анизотропии от нормали к пленке в этой плоскости. На рис. 4 приведена схема, которая иллюстрирует трансформацию распределений поля однородного резонанса при изменении ориентации $\mathbf{H}$ от направления [110] (нормаль к пленке) к направлениям $[1 \overline{1} 0]$ и $[001]$, лежащим в плоскости пленки, а также когда Н изменяет свою ориентацию от [001] к [11̄0].

Используя два набора полей однородного резонанса для соответствующих границ, согласно схеме (рис. 4), с помощью уравнений (1)-(3) были рассчитаны значения эффективных полей анизотропии на свободной поверхности пленки и поверхности, граничащей с подложкой. Как следует из рис. 4 , при изменениях ориентации $\mathbf{H}$ от перпендикулярной к параллельной как в плоскости (001), так и в плоскости (110) происходит смена области локализации нулевой моды от поверхности, граничащей с подложкой к свободной поверхности пленки. На азимутальной зависимости $H_{n}\left(\varphi_{H}\right)$ такого перехода не происходит. При любом $\varphi_{H}$ нулевая мода остается локализованной у свободной поверхности пленки.

Из полученных результатов следует, что при изменении ориентации $\mathbf{H}$ изменяется и величина интервала полей однородного резонанса. Это приводит к изменению $\operatorname{grad} H_{k}^{\text {eff }}$, и как следствие изменению количества возбуждаемых высокоинтенсивных СВ-мод (рис. 2). Расчет спектров спин-волнового резонанса в пленках с однородным градиентом эффективного поля анизотропии позволил установить следующее. Резонансное поле нулевой моды расположено вблизи значения поля однородного резонанса на границе высокополевой области пленки, отклоняясь в область меньших значений примерно на 50-150 Ое. На рис. 5, $a, b$ приведены зависимости относительной интенсивности $I_{n} / I_{0}$

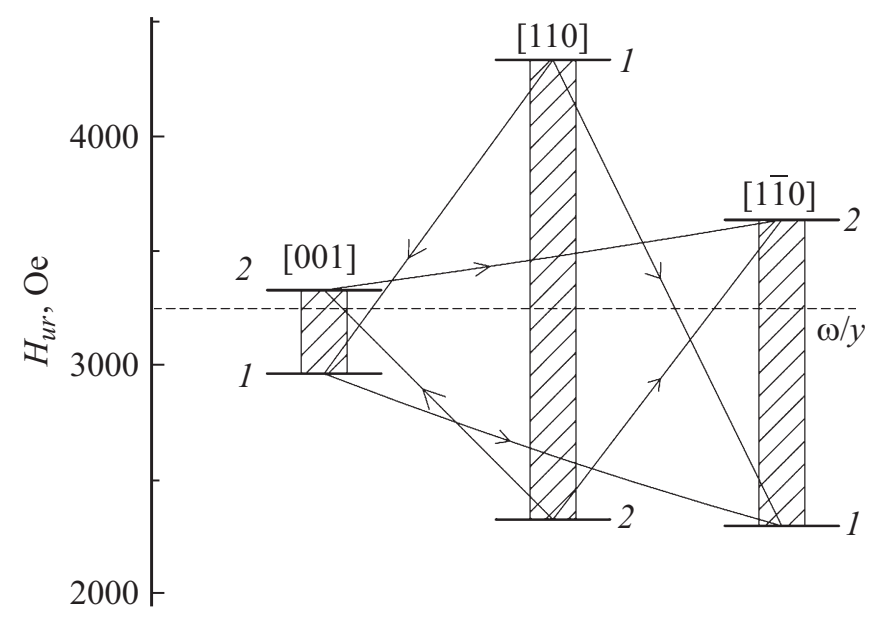

Рис. 4. Диаграмма распределений полей однородного резонанса $H_{u r}$ при различных ориентациях Н относительно пленки. 1 - граница пленки с подложкой, 2 - свободная граница пленки. 

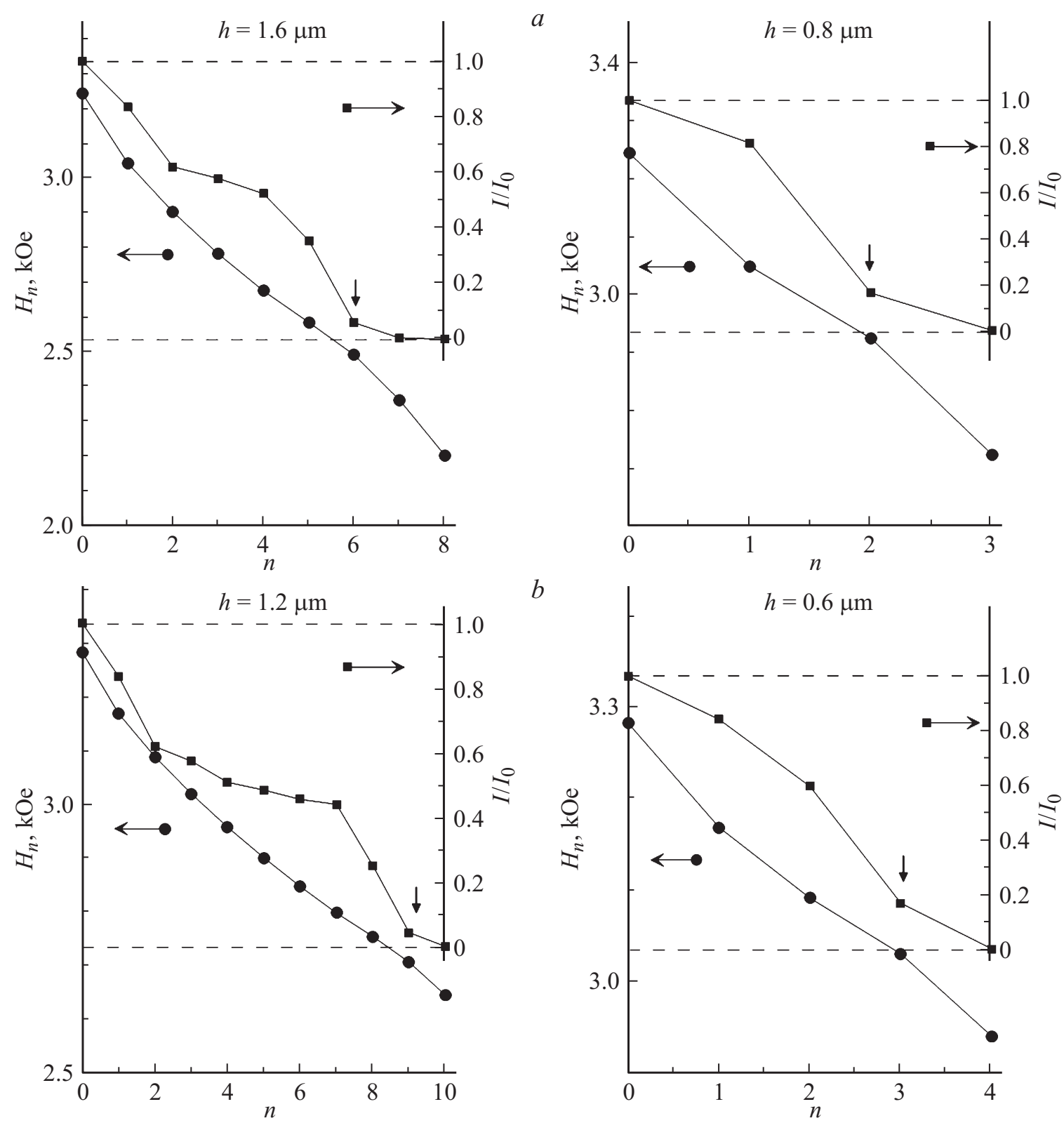

Рис. 5. Расчетные зависимости относительной интенсивности $I_{n} / I_{0}$ и резонансных полей $H_{n}$ СВ-мод от их номера для различных значений толщины пленки $h$ и обменной жесткости $D . a)$ пленка состава $(\mathrm{BiLuGd})_{3}(\mathrm{FeGa})_{5} \mathrm{O}_{12}, D=27 \cdot 10^{-9} \mathrm{erg} \cdot \mathrm{cm}^{-1} \cdot \mathrm{G}^{-1}$; b) $\mathrm{Y}_{3} \mathrm{Fe}_{5} \mathrm{O}_{12}, D=5.3 \cdot 10^{-9} \mathrm{erg} \cdot \mathrm{cm}^{-1} \cdot \mathrm{G}^{-1}$.

и резонансных полей $H_{n}$ возбуждаемых мод от $n$ для пленок с различными значениями толщины $h$ и обменной жесткости $D=(2 A / M)$. Там же штриховыми горизонтальными линиями выделен интервал изменения поля однородного резонанса в пленке. Видно, что по резонансным полям нулевой и последней из высокоинтенсивных СВ-мод можно с некоторой (удовлетворительной) точностью определить поля однородного резонанса на одной и другой границах пленки. При этом идентификацию CВ-моды, точка поворота которой наиболее близка к границе, a eе резонансное поле близко к полю однородного резонанса на этой границе, можно установить по характерному излому (положению точки перегиба) на зависимости относительной интенсивности, а также на зависимости резонансного поля от номера моды $n$. Эти точки на рис. 5 обозначены стрелками. Отметим, что зависимость разности резонансных полей нулевой $n$-й мод хорошо аппроксимируется как $H_{0}-H_{n} \propto(n+1 / 4)^{2 / 3}$. Причем это справедливо только для участка, соответствующего высокоинтенсивным модам.

Результаты, основанные на анализе эволюции спектра $\mathrm{CBP}$, происходящей в результате послойного стравливания пленки, подтверждают корректность вышеизложенного подхода для определения параметров в пленке с однородным градиентом эффективного поля анизотропии. 


\section{5. Выводы}

1. Спектр спин-волнового резонанса в пленках с однородным градиентом эффективного поля анизотропии состоит из серии высокоинтенсивных СВ-мод и мод с малой интенсивностью. В пленках с орторомбической анизотропией на двух из трех характерных угловых зависимостях резонансных полей высокоинтенсивных СВ-мод всегда происходит смена области локализации нулевой моды от одной поверхности пленки к другой. При этом на третьей угловой зависимости область локализации нулевой моды остается неизменной.

2. Показано, что резонансные поля нулевой и последней из высокоинтенсивных СВ-мод лежат вблизи значений полей однородного резонанса на одной и другой поверхностях пленки. По значениям этих полей для трех характерных направлений внешнего магнитного поля $\mathbf{H}$ в неоднородной пленке с орторомбической анизотропией можно определить эффективные поля ромбической и одноосной компонент анизотропии на поверхностях такой пленки. При известном значении гиромагнитного отношения можно определить и поле кубической анизотропии.

3. Установлено, что уменьшение интервала изменения $H_{u r}$ (величины $\left.\operatorname{grad} H_{k}^{\text {eff }}\right)$, который зависит в том числе и от ориентации $H$ относительно осей анизотропии в пленке, приводит к уменьшению количества возбуждаемых высокоинтенсивных СВ-мод и уменьшению разности резонансных полей между ними.

\section{Список литературы}

[1] T. Schneider, A.A. Serga, B. Leven. Appl. Phys. Lett. 92, 022505-1-3 (2008).

[2] Y. Kajiwara, K. Harii, S. Takahashi. Nature 464, 262 (2010).

[3] A. Khitun, M. Bao, K.L. Wang. IEEE Trans. Mag. 44, 2141 (2008).

[4] А.М. Зюзин, С.Н. Сабаев, В.В. Радайкин, А.В. Куляпин. ФTT 44, 893 (2002).

[5] А.М. Зюзин, М.А. Бакулин, Д.А. Зюзин, С.Н. Сабаев. ЖТФ 81, 6, 63 (2011).

[6] B. Hoekstra, R.P. van Stapele, J.M. Robertson. J. Appl. Phys. B 48, 382 (1977).

[7] А.Л. Сукстанский, Г.И. Ямпольский. ФТТ 42, 866 (2000).

[8] А.М. Зюзин, С.Н. Сабаев, А.В. Куляпин. ФТТ 45, 2208 (2003).

[9] А.М. Зюзин, А.Г. Бажанов, В.В. Радайкин. ЖТФ 69, 11, 97 (1999).

[10] X. Liu, Y.Y. Zhou, J.K. Furdyna. Phys. Rev. B 75, 195220 (2007).

[11] А.М. Зюзин, А.Г. Бажанов. Письма в ЖЭТФ 63, 528 (1996).

[12] C. Wilts, S. Prasad. IEEE Trans. Mag. 17, 2405 (1981).

[13] H. Makino, Y. Hidaka. Mater. Res. Bull. 16, 957 (1981).

[14] А.Г. Гуревич, Г.А. Мелков. Магнитные колебания и волны. Физматлит, М. (1994). 464 с.

[15] П.Е. Зильберман, А.В. Луговский, А.А. Шарафатдинов. ФTT 37, 2010 (1995). 